\section{Não adesão à terapia biológica em pacientes com doenças reumáticas no Sistema Único de Saúde em Minas Gerais, Brasil}

\author{
Non-adherence to biological therapy in patients \\ with rheumatic diseases in the Brazilian \\ Unified National Health System in \\ Minas Gerais State, Brazil
}

\section{Falta de adherencia a la terapia biológica de los pacientes con enfermedades reumáticas en el Sistema Único de Salud de Minas Gerais, Brasil}

\begin{abstract}
This study examined non-adherence to biological therapy with adalimumab and etanercept and associated factors in patients with rheumatic diseases treated under the Brazilian Unified National Health System in Minas Gerais State, Brazil. A non-concurrent cohort study was performed from pharmacy records of adult patients that had recently initiated treatment. $\mathrm{Pa}$ tient non-adherence was measured by proportion of days covered (PDC), and non-adherent patients were defined as $P D C<0.8$. A total of 1,150 patients were included, with the following breakdown: $64.3 \%$ rheumatoid arthritis, 8.5\% psoriatic arthritis, and $27.2 \%$ ankylosing spondylitis. In total, 33.5\% of patients were nonadherent. Factors such as younger age (19-39 years), female gender, diagnosis of rheumatoid arthritis, and initial treatment with etanercept were associated with low adherence. The results indicate a high prevalence of non-adherence, which can lead to worse health outcomes and increase healthcare use and costs.
\end{abstract}

Medication Adherence; Biological Therapy; Rheumatic Diseases
Clarice Gomes e Souza Dabés 1 Alessandra Maciel Almeida 1,2 Francisco de Assis Acurcio ${ }^{1}$

\section{Resumo}

Neste estudo, buscou-se estimar e analisar fatores associados à não adesão ao tratamento com os medicamentos biológicos adalimumabe e etanercepte em pacientes portadores de doenças reumáticas atendidos pelo Sistema Único de Saúde de Minas Gerais, Brasil. Procedeu-se a estudo prospectivo não concorrente com base no registro de dispensação nas farmácias de pacientes adultos em início de tratamento. A taxa de não adesão foi estimada com utilização do método de proporção de dias cobertos (PDC), sendo considerados não aderentes pacientes com $P D C<0$,8. Foram identificados 1.150 pacientes, sendo $64,3 \%$ portadores de artrite reumatoide, 8,5\% de artrite psoriásica e 27,2\% de espondilite anquilosante. A taxa de não adesão encontrada foi de 33,5\% e os fatores associados foram idade entre 19-39 anos, sexo feminino, diagnóstico de artrite reumatoide e início de tratamento com etanercepte. Os resultados indicam uma alta taxa de não adesão ao tratamento, que pode originar piores resultados em saúde e implicar o aumento da utilização de cuidados e dos custos em saúde.

Adesão à Medicação; Terapia Biológica; Doenças Reumáticas 


\section{Introdução}

Artrite reumatoide, artrite psoriásica e espondilite anquilosante são doenças autoimunes inflamatórias crônicas associadas à destruição e deformidades das articulações. A prevalência mundial da artrite reumatoide é de 0,4 a 1,9\%. No Brasil, existem poucos estudos de prevalência, mas estima-se que seja entre 0,2 e $1 \% 1,2$. Acomete predominantemente mulheres entre 30-50 anos. A artrite psoriásica apresenta prevalência mundial bastante variada, podendo chegar a 0,42\%. Em mais da metade dos casos é consequência da psoríase de longa duração ${ }^{3}$. A espondilite anquilosante possui prevalência de 0,1 a 0,4\% em diversos países, tendo associação significativa com o antígeno de histocompatibilidade (antígeno leucocitário humano) HLA B27 4. É predominante no sexo masculino entre a segunda e quarta décadas de vida.

O tratamento dessas doenças inclui abordagens não medicamentosas e medicamentosas. Entre os medicamentos utilizados, há os que controlam apenas os sintomas e os que atuam diretamente no processo da doença, chamados de medicamentos modificadores do curso da doença (MMCD). Esses MMCD podem ser sintéticos ou biológicos, e o advento dos últimos mudou de forma significativa o tratamento das doenças reumáticas, uma vez que auxiliaram na diminuição do processo inflamatório e na inibição do dano estrutural progressivo 5 .

Os MMCD sintéticos e biológicos são fornecidos, gratuitamente, pelo Sistema Único de Saúde (SUS). O primeiro medicamento biológico introduzido no elenco de medicamentos foi o infliximabe, em 2002, para o tratamento da artrite reumatoide. Em 2006, adalimumabe e etanercepte passaram a ser fornecidos também para essa doença. Para o tratamento da artrite psoriásica e da espondilite anquilosante, os três biológicos (adalimumabe, etanercepte e infliximabe) tornaram-se disponíveis em 2010. Esses medicamentos são da classe dos inibidores do fator de necrose tumoral (anti-TNF). Em 2013, foram incluídos mais dois biológicos anti-TNF (certolizumabe e golimumabe), além do abatacepte, tocilizumabe e rituximabe.

Atualmente, as regras de financiamento, aquisição e fornecimento pelo SUS de medicamentos biológicos são regulamentadas por meio do Componente Especializado da Assistência Farmacêutica (CEAF). A aquisição é feita de maneira centralizada pelo Ministério da Saúde e a dispensação é realizada pelas esferas estaduais. As linhas de tratamento são definidas em Protocolos Clínicos e Diretrizes Terapêuticas (PCDT), sendo necessário que o paciente atenda aos cri- térios de inclusão estabelecidos em tais protocolos para que recebam o medicamento. O PCDT para o tratamento da artrite reumatoide está disponível desde 2002. Para artrite psoriásica e espondilite anquilosante, que não possuíam PCDT homologados, as linhas de tratamento eram definidas no âmbito estadual.

Para atingir resultados desejáveis com a terapia anti-TNF, assim como em outras doenças crônicas, a adesão do paciente ao tratamento é fundamental ${ }^{6}$. A baixa adesão traz consequências negativas para o tratamento farmacológico das doenças reumáticas, uma vez que os MMCD reduzem a atividade da doença e a progressão radiológica, além de melhorar os resultados funcionais em longo prazo 7 .

Segundo a Organização Mundial da Saúde (OMS), diversos fatores podem influenciar a adesão, como fatores socioeconômicos, relacionados à equipe e ao sistema de saúde, à condição, à terapia e ao paciente ${ }^{8}$. Neste estudo, buscou-se estimar a proporção de pacientes não aderentes à terapia biológica, por meio de medida indireta, bem como identificar possíveis fatores associados a esse evento.

\section{Métodos}

Trata-se de um estudo prospectivo não concorrente (coorte histórica) que tem como fonte de dados os registros de dispensação obtidos no Sistema de Informação e Gestão Hospitalar (SIGH) utilizado pela Secretaria de Estado de Saúde de Minas Gerais (SES/MG) para atendimento aos usuários.

Foram incluídos no estudo todos os pacientes atendidos com medicamentos biológicos, em Minas Gerais, no período de 1o de janeiro a 31 de dezembro de 2011. Durante esse período, os medicamentos biológicos disponíveis pelo SUS eram adalimumabe, etanercepte e infliximabe. Foi considerado que todos os pacientes incluídos atendiam aos critérios de diagnóstico do PCDT para artrite reumatoide e aos critérios estabelecidos em âmbito estadual para artrite psoriásica e espondilite anquilosante, tendo em conta que não há PCDT para essas doenças, e que esses são pré-requisitos para que o paciente receba os medicamentos pela SES/MG.

Foram excluídos os pacientes com diagnóstico diferente de artrite reumatoide, artrite psoriásica ou espondilite anquilosante (conforme a 10a revisão da Classificação Internacional de Doenças - CID-10: M05.0, M05.1, M05.2, M05.3, M05.8, M06.0 e M06.8; M07.0 e M07.3; M45); pacientes com idade inferior a 18 anos; e pacientes que utilizaram qualquer MMCD biológico nos 
365 dias anteriores à data do primeiro registro de dispensação. Posteriormente, pacientes que receberam infliximabe durante o período também foram excluídos devido às inconsistências dos dados, que impossibilitaram a avaliação do uso de cada dose. Nesse caso, a quantidade de frascos fornecidos não correspondeu exatamente à dose efetivamente aplicada e nem ao período da aplicação. Os frascos fornecidos pelo serviço eram de 100mg e não eram fracionáveis. A dose preconizada no início de tratamento com infliximabe é de $3 \mathrm{mg} / \mathrm{kg}$ de peso nas semanas 0,2 e 6, e após a cada oito semanas ${ }^{9}$. Dessa forma, os registros da farmácia apresentavam o número total de frascos fornecidos por um período, mas não foi possível extrair os dados necessários para o cálculo da adesão ao infliximabe.

Pacientes que trocaram de medicamento biológico dentro do período de 12 meses foram excluídos do estudo por conta da existência de intervalos, sem registro de dispensação, entre o primeiro e o segundo medicamento, superiores a 70 dias. Esse intervalo poderia classificar o paciente como não aderente ao tratamento, e o tempo sem medicamento pode ter sido por causa do trâmite administrativo para a troca do medicamento.

Os registros de dispensação de todos os pacientes incluídos foram obtidos no software SIGH (Companhia de Processamentos de Dados do Estado de São Paulo, São Paulo, Brasil), sendo cada paciente acompanhado por um período de 365 dias. Esse software é utilizado na SES/MG e está presente em 26 das 28 regionais de saúde do Estado de Minas Gerais. As regionais de Barbacena e Manhumirim possuíam registros manuais no período do estudo e, portanto, foram excluídas do estudo.

O desfecho avaliado foi a taxa de não adesão, considerando o monitoramento da posse do medicamento, estimada pela medida indireta proporção de dias cobertos (PDC) ${ }^{10}$. Essa opção se baseou nas características do fornecimento de medicamento e agendamento dos pacientes do CEAF em Minas Gerais. No caso de fornecimento antecipado do medicamento, os dias de antecipação foram considerados para o agendamento do próximo atendimento.

A PDC foi calculada pela soma da quantidade de dias em que o paciente ficou em posse do medicamento durante um período fixo de 365 dias, sendo seguidas as seguintes etapas 10,11:

1) Foi determinado o período de seguimento do paciente, que foi definido como os 365 dias subsequentes à data da primeira prescrição do biológico. A data da primeira dispensação de medicamento biológico constituiu a linha de base do estudo.
2) Dentro do período de acompanhamento, contaram-se os dias em que o paciente foi coberto pelo medicamento baseado na data de atendimento da prescrição e dias de fornecimento. Caso a prescrição sobrepusesse o abastecimento anterior, em seguida, a data de início da prescrição era ajustada como o dia após o fim do abastecimento anterior.

3) Dividiu-se o número de dias em que o paciente foi coberto por medicamento pelo período de seguimento.

Para todas as doenças foram considerados aderentes os pacientes que apresentaram PDC $\geq 0,80$ e não aderentes aqueles com $\operatorname{PDC}<0,80$. O ponto de corte de 0,80 foi comumente utilizado em estudos de adesão de medicamentos biológicos utilizados no tratamento da artrite reumatoide 12,13,14.

Os dias de posse com o medicamento foram calculados pela quantidade dispensada, tendo como referência as doses preconizadas no PCDT (artrite reumatoide), nos consensos (artrite psoriásica e espondilite anquilosante) e também a quantidade mensal de medicamento autorizada pelo SUS ${ }^{15}$. As apresentações farmacêuticas disponibilizadas pela SES/MG foram: seringas de adalimumabe $40 \mathrm{mg}$; etanercepte $25 \mathrm{mg}$ e $50 \mathrm{mg}$. A dose preconizada para uso do adalimumabe é de 40mg a cada duas semanas e do etanercepte é de 50mg semanalmente. Dessa forma, duas seringas de adalimumabe de $40 \mathrm{mg}$, oito seringas de etanercepte $25 \mathrm{mg}$ ou quatro seringas de etanercepte de 50mg forneceram 28 dias de posse do medicamento.

As variáveis independentes foram sexo, idade, medicamento no início do tratamento, doença e Índice de Desenvolvimento Humano do Município (IDH-M) de residência.

A análise descritiva dos dados foi realizada por meio de medidas de tendência central para variáveis contínuas e medidas de frequência para as categóricas. As diferenças de proporção foram analisadas pelo teste qui-quadrado de Pearson. Regressão logística foi utilizada para avaliar o efeito independente das variáveis explicativas, comparando os grupos aderentes e não aderentes. Nessa análise, a idade foi categorizada segundo os quartis de sua distribuição e o IDH-M categorizado em dois grupos, em função da mediana. Considerou-se, como critério de inclusão das variáveis independentes no modelo inicial, um valor de $\mathrm{p} \leq 0,15$ na análise bivariada, com a exclusão sequencial de variáveis sem efeito independente significativo. A estatística de HosmerLemeshow foi utilizada para análise de adequação do modelo logístico final. Todas as análises adotaram o nível de significância de 0,05. Os dados foram trabalhados utilizando o software phpMy 
Admin (https://www.phpmyadmin.net/). Foi utilizado o programa estatístico $\mathrm{R}$ versão 3.0.1 (The R Foundation for Statistical Computing, Viena, Áustria; http://www.r-project.org).

Este estudo é parte integrante do projeto de pesquisa Avaliação da Efetividade e Segurança dos Anticorpos Monoclonais Adalimumabe, Etanercepte, Infliximabe e Rituximabe Utilizados no Tratamento da Artrite Reumatoide, Artrite Psoriática e Espondilite Anquilosante, Brasil e Minas Gerais, aprovado no Comitê de Ética em Pesquisa da Universidade Federal de Minas Gerais em 26 de maio de 2011 (parecer no ETIC 0069.0.203.000-11).

\section{Resultados}

Em 2011, 3.529 pacientes receberam, por pelo menos uma vez, os medicamentos biológicos adalimumabe, etanercepte e/ou infliximabe. Após aplicação dos critérios de exclusão de doença e idade, 2.967 pacientes foram elegíveis para o estudo. Desses, 1.687 foram excluídos, pois tiveram registro de dispensação de biológico nos 365 dias anteriores ao primeiro atendimento no ano de 2011. Depois foram excluídos os pacientes que receberam infliximabe $(n=106)$ durante o período e os que trocaram de medicamento biológico $(n=24)$, resultando em uma amostra com 1.150 pacientes (Figura 1).

Do total de 1.150 pacientes, 739 (64,3\%) eram portadores de artrite reumatoide, 98 (8,5\%) de artrite psoriásica e $313(27,2 \%)$ de espondilite anquilosante. Os pacientes com artrite reumatoide eram mais velhos que os demais, sendo as medianas das idades de 52,0; 50,5 e 40,0 anos para artrite reumatoide, artrite psoriásica e espondilite anquilosante, respectivamente $(\mathrm{p}<0,001$ Tabela 1).

Em relação à distribuição por sexo, a maioria dos pacientes portadores de artrite reumatoide eram mulheres (84\%), enquanto para artrite psoriásica e espondilite anquilosante o sexo feminino correspondeu a $42,9 \%$ e $32,9 \%$ dos pacientes respectivamente.

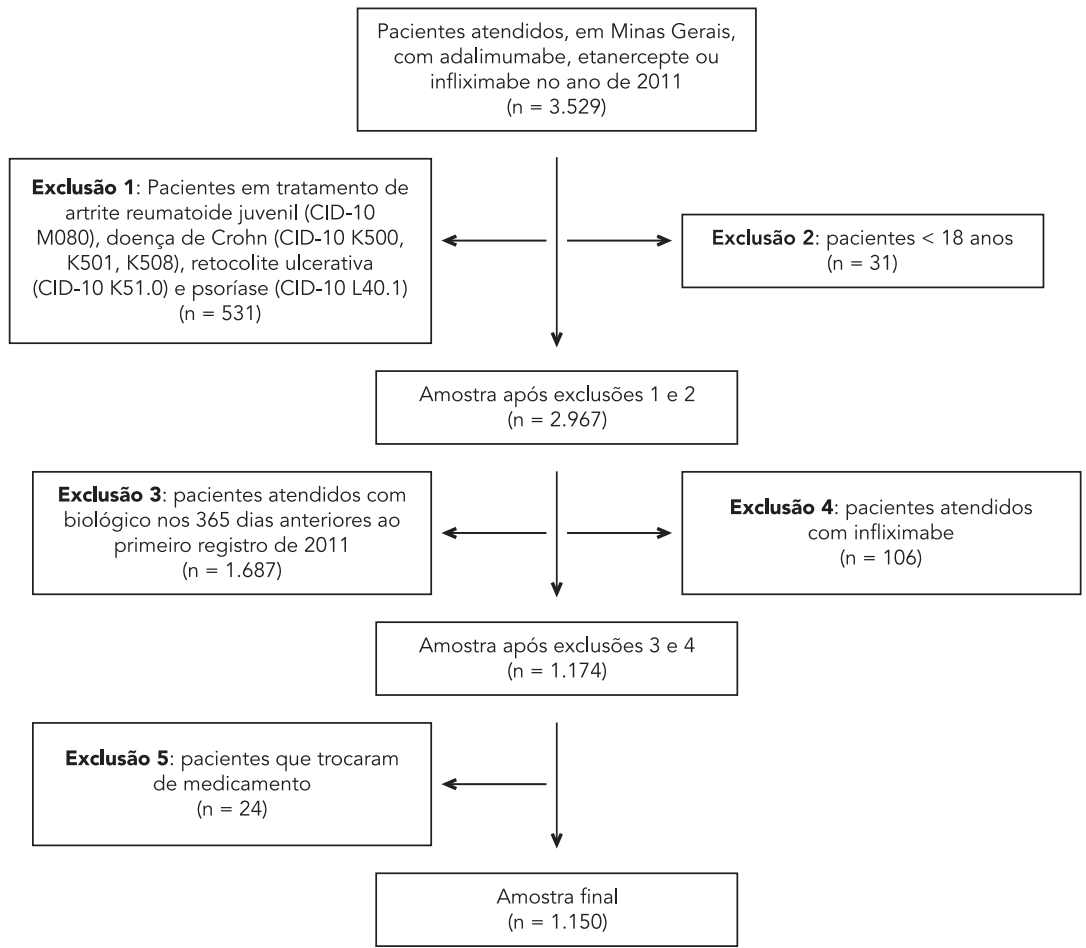

CID-10: Classificação Internacional de Doenças, 10ạ revisão. 
Características dos pacientes que iniciaram o uso de medicamentos biológicos no ano de 2011 por doença. Sistema Único de Saúde (SUS), Minas Gerais, Brasil.

\begin{tabular}{|c|c|c|c|c|c|}
\hline \multirow[t]{2}{*}{ Característica } & \multicolumn{4}{|c|}{ Número de pacientes (\%) } & \multirow[t]{2}{*}{ Valor de $p$} \\
\hline & Artrite reumatoide & Artrite psoriásica & $\begin{array}{l}\text { Espondilite } \\
\text { anquilosante }\end{array}$ & Total & \\
\hline Total & $739(64,3)$ & $98(8,5)$ & $313(27,2)$ & 1.150 & \\
\hline \multicolumn{6}{|l|}{ Idade (anos) } \\
\hline Média $\pm D P$ & $52,1 \pm 13,3$ & $49,8 \pm 14,2$ & $41,0 \pm 11,5$ & $48,9 \pm 13,8$ & \multirow{2}{*}{$<0,001$ * } \\
\hline Mediana & 52,0 & 50,5 & 40,0 & 49,0 & \\
\hline \multicolumn{6}{|l|}{ Sexo } \\
\hline Feminino & $621(84,0)$ & $42(42,9)$ & $103(32,9)$ & $766(66,6)$ & \multirow{2}{*}{$<0,001$ ** } \\
\hline Masculino & $118(16,0)$ & $56(57,1)$ & $210(67,1)$ & $384(33,4)$ & \\
\hline \multicolumn{6}{|l|}{ Medicamento } \\
\hline Adalimumabe & $418(56,6)$ & $48(49,0)$ & $155(49,5)$ & $621(54,0)$ & \multirow{2}{*}{$0,065 * *$} \\
\hline Etanercepte & $321(43,4)$ & $50(51,0)$ & $158(50,5)$ & $529(46,0)$ & \\
\hline \multicolumn{6}{|l|}{ IDH-M } \\
\hline 0,$587 ; 0,713$ & $178(24,1)$ & $17(17,3)$ & $98(31,3)$ & $293(25,1)$ & \multirow{4}{*}{$0,022 * \star$} \\
\hline 0,$713 ; 0,756$ & $202(27,3)$ & $23(23,5)$ & $64(20,4)$ & $289(25,1)$ & \\
\hline 0,$756 ; 0,778$ & $186(25,2)$ & $27(27,6)$ & $82(26,2)$ & $295(25,7)$ & \\
\hline 0,$778 ; 0,813$ & $173(23,4)$ & $31(31,6)$ & $69(22,1)$ & $273(23,7)$ & \\
\hline
\end{tabular}

DP: desvio padrão; IDH-M: Índice de Desenvolvimento Humano Municipal.

* Teste de Kruskal-Wallis;

** Teste de qui-quadrado.

O medicamento adalimumabe foi utilizado no início do tratamento por mais da metade dos pacientes $(54 \% ; \mathrm{n}=621)$, e entre os portadores de atrite reumatoide o uso foi de $56,6 \%$. Nas demais doenças, o percentual de pacientes que iniciou o tratamento com adalimumabe foi semelhante ao etanercepte.

Quando se observou a distribuição dos pacientes entre as diferentes doenças por IDH-M, verificou-se que há uma tendência de aumento do número de pacientes com artrite psoriásica quanto maior o índice.

A proporção de pacientes não aderentes ao tratamento foi de $33,5 \%(\mathrm{n}=385)$. No modelo univariado, verificou-se que há diferença significativa na taxa de não adesão quando se avalia sexo, doença e medicamento ( $p<0,05$; Tabela 2). Comparando-se os sexos, a proporção de pacientes não aderentes foi maior entre as mulheres do que entre os homens $(36,6 \%$ versus $27,3 \% ; p=0,002$ ). Também foi identificada maior proporção de não aderentes entre aqueles que iniciaram o tratamento com etanercepte (38\%) quando comparados aos que iniciaram com adalimumabe $(29,6 \% ; p=0,003)$. Em contrapartida, os pacientes com artrite psoriásica e espondilite anquilosante apresentaram menor taxa de não adesão quando comparados aos pacientes com artrite reumatoide (taxa de não adesão de 21,4\% e $28,4 \%$, respectivamente, versus $37,2 \%$; $\mathrm{p}<0,05$ ). A taxa de não adesão foi semelhante quando se comparou as faixas etárias e o IDH-M da residência dos pacientes.

A análise multivariada, por meio de regressão logística, avaliou a possível associação de fatores como idade, sexo, doença, medicamento e IDH-M com a taxa de não adesão. As variáveis idade, sexo, doença e medicamento foram associadas à não adesão no modelo final $(\mathrm{p}<0,05)$ (Tabela 3).

Pacientes com idade entre 50-58 anos tiveram menos chance de não adesão $(\mathrm{OR}=0,65$; $\mathrm{p}=0,020)$ do que pacientes na faixa etária de 1939 anos. As mulheres apresentaram 1,37 vez mais chance de não aderirem ao tratamento que os homens $(p=0,048)$. A análise mostrou que os pacientes diagnosticados com artrite reumatoide tiveram mais chance de não aderirem ao tratamento quando comparados aos portadores de artrite psoriásica (OR = 0,48; IC95\%: 0,49-0,83) e de espondilite anquilosante $(\mathrm{OR}=0,71$; IC95\%: 0,50-0,99). Quando se avaliou o medicamento no início do tratamento, o uso inicial de etanercepte se relacionou com uma chance maior de 
Análise univariada da associação de variáveis selecionadas e taxa de não adesão ao tratamento com medicamentos biológicos. Minas Gerais, Brasil, 2011-2012.

\begin{tabular}{|c|c|c|c|c|c|}
\hline \multirow[t]{2}{*}{ Variável } & \multicolumn{5}{|c|}{ Não aderentes (PDC < 0,8) } \\
\hline & $\mathbf{n}$ & $\%$ & OR bruta & IC95\% & Valor de $p$ \\
\hline Total $[\mathrm{N}=1.150]$ & 385 & 33,5 & - & - & - \\
\hline \multicolumn{6}{|l|}{ Idade } \\
\hline 1ㅇ quartil (19-39 anos) [n=311] & 107 & 34,4 & 1,00 & - & - \\
\hline 2o quartil (40-49 anos) [ $n=268]$ & 91 & 34,0 & 0,98 & $0,69-1,38$ & 0,909 \\
\hline 3o quartil (50-58 anos) [ $n=296]$ & 86 & 29,1 & 0,78 & $0,55-1,10$ & 0,157 \\
\hline 4o quartil (59-90 anos) [ $n=275]$ & 101 & 36,7 & 1,11 & $0,79-1,55$ & 0,558 \\
\hline \multicolumn{6}{|l|}{ Sexo } \\
\hline Masculino $[\mathrm{n}=384]$ & 105 & 27,3 & 1,00 & - & - \\
\hline Feminino $[n=766]$ & 280 & 36,6 & 1,53 & $1,17-2,00$ & 0,002 * \\
\hline \multicolumn{6}{|l|}{ Doença } \\
\hline Artrite reumatoide $[n=739]$ & 275 & 37,2 & 1,00 & - & - \\
\hline Artrite psoriásica [n = 98] & 21 & 21,4 & 0,46 & $0,27-0,76$ & 0,003 * \\
\hline Espondilite anquilosante $[n=313]$ & 89 & 28,4 & 0,67 & $0,50-0,89$ & 0,006 * \\
\hline \multicolumn{6}{|l|}{ Medicamento } \\
\hline Adalimumabe $[n=621]$ & 184 & 29,6 & 1,00 & - & - \\
\hline Etanercepte $[n=529]$ & 201 & 38,0 & 1,46 & $1,14-1,86$ & 0,003 * \\
\hline \multicolumn{6}{|l|}{ IDH-M } \\
\hline 0,$587 ; 0,756[n=582]$ & 193 & 33,2 & 1,00 & - & - \\
\hline 0,$756 ; 0,813[n=568]$ & 192 & 32,7 & 1,03 & $0,81-1,31$ & 0,817 \\
\hline
\end{tabular}

IC95\%: intervalo de 95\% de confiança; IDH-M: Índice de Desenvolvimento Humano Municipal; OR: odds ratio;

PDC: proporção de dias cobertos.

* Valor de $\mathrm{p}<0,05$

não adesão em comparação aos pacientes que iniciaram o uso de adalimumabe $(\mathrm{OR}=1,54$; IC95\%: 1,20-1,98).

\section{Discussão}

Este estudo possibilitou descrever as características dos pacientes que iniciaram o tratamento com medicamentos biológicos, para as doenças reumáticas artrite reumatoide, artrite psoriásica e espondilite anquilosante, atendidos pelo SUS de Minas Gerais em 2011. Também permitiu estimar a proporção de pacientes não aderentes ao tratamento e identificar possíveis fatores associados a esse evento.

As características demográficas dos pacientes foram semelhantes às observadas na literatura 5,16,17. Na artrite reumatoide houve predominância do sexo feminino e a idade média foi superior à das demais doenças. Ao contrário, pacientes com espondilite anquilosante eram predominantemente do sexo masculino e mais jovens. Os pacientes com artrite psoriásica não apresentaram predominância de sexo e apresentaram média de idade entre a quarta e quinta década de vida.

Em relação à distribuição do medicamento, considerando os medicamentos disponibilizados pelo SUS para o tratamento das doenças reumáticas no período do estudo, o adalimumabe foi utilizado por mais da metade dos pacientes portadores de artrite reumatoide. Essa maior utilização do adalimumabe também foi encontrada em outros estudos brasileiros 18,19. Um estudo sobre demandas por medicamentos via judicial, conduzido em Minas Gerais, relatou que, entre os anos de 1999 e 2009, o adalimumabe foi o medicamento mais prescrito 20 . O ano de 2006 foi destaque de crescimento da demanda por esse medicamento. O estudo sugere a influência da indústria farmacêutica na maior frequência de prescrição desse medicamento, envolvendo médicos prescritores e escritórios de advocacia.

Analisando a distribuição das doenças, a maioria dos pacientes era portadora de artrite reumatoide, seguida de espondilite anquilosante e artrite psoriásica. A maior prevalência de pa- 
Modelo de regressão logística para análise da não adesão ao tratamento de doenças reumáticas com biológicos em pacientes no início do tratamento. Minas Gerais, Brasil, 2011-2012.

\begin{tabular}{|c|c|c|c|}
\hline Variável & OR & IC95\% & Valor de $\mathrm{p}$ \\
\hline \multicolumn{4}{|l|}{ Idade } \\
\hline 1ㅇ quartil (19-39 anos) [n=311] & 1,00 & - & - \\
\hline 2o quartil (40-49 anos) [n = 268] & 0,87 & $0,61-1,24$ & 0,429 \\
\hline 3o quartil (50-58 anos) [n = 296] & 0,65 & $0,45-0,93$ & 0,020 * \\
\hline 4o quartil (59-90 anos) [ $n=275]$ & 0,86 & $0,60-1,24$ & 0,422 \\
\hline \multicolumn{4}{|l|}{ Sexo } \\
\hline Masculino $[n=384]$ & 1,00 & - & - \\
\hline Feminino $[n=766]$ & 1,37 & $1,00-1,87$ & 0,048 * \\
\hline \multicolumn{4}{|l|}{ Doença } \\
\hline Artrite reumatoide $[n=739]$ & 1,00 & - & - \\
\hline Artrite psoriásica [n = 98] & 0,48 & $0,49-0,83$ & 0,008 * \\
\hline Espondilite anquilosante [ $\mathrm{n}=313]$ & 0,71 & $0,50-0,99$ & 0,048 * \\
\hline \multicolumn{4}{|l|}{ Medicamento no início do tratamento } \\
\hline Adalimumabe $[\mathrm{n}=621]$ & 1,00 & - & - \\
\hline Etanercepte [n = 529] & 1,54 & $1,20-1,98$ & $<0,001 *$ \\
\hline
\end{tabular}

IC95\%: intervalo de 95\% de confiança; OR: odds ratio.

* Valor de $\mathrm{p}<0,05$.

Nota: Hosmer-Lemeshow: $p=0,966$.

cientes com espondilite anquilosante em comparação com a artrite psoriásica também foi observada por Gallinaro et al. 21, que compararam dados de um estudo epidemiológico brasileiro com os dados de diversos países ibero-americanos. Diferentemente, Bakland \& Nossent 22 encontraram que a prevalência de artrite psoriásica foi semelhante à da espondilite anquilosante em diversas regiões do mundo na população em geral. A incidência e a prevalência da espondilite anquilosante podem estar associadas à prevalência do HLA B-27 na população.

A taxa de não adesão à terapia biológica verificada neste estudo foi alta; um terço dos pacientes não aderiu ao tratamento no primeiro ano. Comparando-se os resultados de outros estudos que também utilizaram dados administrativos, as taxas de não adesão variaram entre $25 \%$ e $68 \%$ $6,11,12,13,23$. Contudo, avaliaram apenas pacientes portadores de artrite reumatoide. Uma das limitações em estudos que avaliam a não adesão diz respeito à grande variação de métodos para cálculo das medidas de adesão, o que dificulta a comparabilidade entre os estudos. Além disso, poucos estudos avaliaram os MMCD biológicos adalimumabe e etanercepte 6,12,23. Em uma revisão sistemática que incluiu estudos com pacientes em uso de biológicos anti-TNF para o trata- mento da artrite reumatoide 24 , foi encontrada uma taxa semelhante de não adesão, ou seja, de um terço. O estudo de Li et al. 11 foi o que encontrou maior taxa de não adesão em pacientes portadores de artrite reumatoide, sendo de $68 \%$ para o etanercepte. Os autores sugerem que o fato pode estar relacionado com a característica da população estudada, que era de baixo poder aquisitivo. Os estudos que utilizaram fonte de dados administrativos e avaliaram a adesão ao adalimumabe e/ou etanercepte apresentaram resultados semelhantes a este estudo 12,13.

Além do uso de bases de dados administrativos, outros métodos também foram utilizados para estimar a adesão, como o uso de questionários autorrelatos, registros de uso de medicamento, opinião do médico e uso de dispositivos eletrônicos. Limitações como diferenças metodológicas, não inclusão dos medicamentos biológicos, não discriminação dos resultados por classe de medicamento, tempos diferentes de acompanhamento e diferenças quanto aos critérios de inclusão também dificultaram a comparabilidade entre os estudos.

Para a artrite psoriásica, não foram encontrados estudos que avaliaram a adesão ao tratamento com medicamentos biológicos. Na espondilite anquilosante, Arturi et al. 25 , utilizando o ques- 
tionário de avaliação da adesão em reumatologia, observaram alta taxa de não adesão ao tratamento $(74,6 \%)$. Porém, foram avaliadas outras classes de medicamentos além dos biológicos, e apenas $35,6 \%$ dos pacientes estavam em uso desses medicamentos.

Os fatores associados à não adesão ao tratamento com biológicos encontrados no presente estudo foram: faixa etária de 19-39 anos (comparada à faixa etária de 50-58 anos), sexo feminino, diagnóstico de artrite reumatoide e início de tratamento com etanercepte. O $3^{\circ}$ quartil da faixa etária, de 50 a 58 anos, apresentou menor proporção de pacientes não aderentes que o $1^{\circ}$ quartil, de 19 a 39 anos. Na literatura, a idade tem relação divergente com a adesão. $\mathrm{O}$ único estudo com medicamento biológico que encontrou relação de adesão com a idade foi conduzido por Li et al. 11, sendo encontrada maior proporção de pacientes aderentes na faixa etária de 55-64 anos nos pacientes de artrite reumatoide, semelhante à proporção encontrada neste estudo. Em outros estudos com medicamentos não biológicos foi apontada a ocorrência de maior adesão entre os mais velhos, assim como em outros a idade não foi associada de forma significante ao evento 26 .

Fidder et al. ${ }^{24}$, em revisão sistemática de adesão a medicamentos biológicos na artrite reumatoide, encontraram que o sexo feminino foi o fator mais fortemente associado à ocorrência de não adesão. Curkendall et al. 6 também encontraram associação do sexo feminino à alta taxa de não adesão aos biológicos adalimumabe e etanercepte. As razões para a menor taxa de não adesão entre os homens ainda é desconhecida 27. Entretanto, em uma revisão sistemática que identificou fatores associados à adesão no tratamento da artrite reumatoide 26 , o sexo apresentou associação conflitante. Ressalta-se que tal revisão incluiu estudos que avaliaram tratamento com outros medicamentos além dos biológicos.

Poucos estudos compararam a adesão por agente biológico. Neste estudo, o etanercepte foi relacionado à maior taxa de não adesão. Ao contrário, no estudo de Borah et al. 12, o etanercepte foi associado à menor taxa de não adesão ao tratamento quando comparado ao adalimumabe $(p=0,08)$. Vale ressaltar que na literatura não são encontradas diferenças de eficácia e segurança entre os dois medicamentos 28,29. Revisões sistemáticas demonstraram que o tratamento da arttrite reumatoide com etanercepte está associado a um menor número de interrupções por reações adversas quando comparado ao adalimumabe ${ }^{30}$, mas foi constatada elevada heterogeneidade nas metanálises, que sugerem que esse achado seja interpretado com cautela 31 .
Uma das diferenças entre esses medicamentos é a frequência de administração, sendo o adalimumabe quinzenal e o etanercepte semanal. Regimes de tratamento com menor frequência de doses favorecem a adesão de uma variedade de classes terapêuticas em pacientes portadores de doenças não reumáticas 32 . Estudo realizado com pacientes em uso de biológicos subcutâneos, incluindo o adalimumabe e etanercepte apresentou preferência por tratamentos com intervalos mais longos entre as injeções 33 .

Comparando-se os diagnósticos, a artrite reumatoide esteve relacionada à maior taxa de não adesão. Um estudo que avaliou a adesão ao tratamento da espondilite anquilosante, tendo a artrite reumatoide como grupo controle 25 e utilizando como método um questionário de adesão, encontrou que pacientes com artrite reumatoide foram mais aderentes quando comparados aos pacientes com espondilite anquilosante. Todavia, a adesão foi avaliada para diversas classes de medicamentos além dos biológicos. Os últimos foram utilizados por pouco mais de um terço da população estudada.

Não foram encontrados outros estudos que compararam a não adesão à terapia biológica entre as doenças de interesse. Contudo, há estudos que compararam taxas de retenção ao tratamento, por método de análise de sobrevivência, que atribuíram ao diagnóstico de atrite reumatoide menor retenção em relação às espondiloartrites artrite psoriásica e espondilite anquilosante 17,34,35,36. Duclos et al. 17 abordam, como umas das justificativas, a influência do TNF na fisiopatologia da doença, e esse fator pode ser mais importante nas espondiloartrites do que na artrite reumatoide. Também ressalta que uma população mais jovem com menor uso de corticoides e imunossupressivos poderia ter uma tolerância maior à terapia biológica quando comparada à população com artrite reumatoide. Heiberg et al. 35 acrescentam que a taxa de retenção ao tratamento com anti-TNF pode ser, ainda, influenciada pela disponibilidade de tratamentos alternativos. Para a espondilite anquilosante, a terapia biológica é o único tratamento efetivo recomendado para pacientes com doença axial que falharam com o uso de antiinflamatórios não esteroidais.

O uso de banco de dados administrativos possui limitações importantes; uma delas é que o objetivo dos registros é de autorização/ressarcimento do sistema de saúde e não de coleta de dados para estudos clínico-epidemiológicos. Outra limitação do estudo está relacionada à estimativa da não adesão, que foi realizada por intermédio de um método indireto. A PDC é avaliada como uma das principais medidas indiretas de ade- 
são 37,38 . No entanto, o registro do fornecimento do medicamento não indica indubitavelmente que o paciente o utilizou, mas sim que o medicamento ficou disponível para uso durante determinado período. Por outro lado, se a PDC implica uma disponibilidade de medicamentos inferior à quantidade mínima considerada suficiente para a adesão à terapia, é adequado se utilizar o registro de dispensação para estimar a ocorrência de não adesão. Também não foram investigados quaisquer resultados na saúde decorrentes dessa utilização nem as razões de não adesão. Dessa forma, este estudo não reflete efetividade clínica, mas propicia uma melhor compreensão da ocorrência de não adesão entre os pacientes com doenças reumáticas. Outros fatores relacionados ao paciente e à condição, como crenças e expectativas dos pacientes, gravidade dos sintomas, índices de atividade da doença e perda de capacidade, que podem contribuir para a não adesão ao tratamento, não foram avaliados 7,35.

\section{Considerações finais}

Este estudo contribui para o conhecimento acerca do uso dos medicamentos biológicos no âmbito do SUS em Minas Gerais. Foi descrito o perfil dos pacientes em uso desses medicamentos na artrite reumatoide, artrite psoriásica e espondilite anquilosante, em 2011.
A taxa de não adesão encontrada foi relativamente alta, assim como também é encontrada em estudos realizados em outros países. Embora a não adesão tenha sido associada com características sociodemográficas, à doença e ao medicamento, outros fatores devem ser investigados de forma a ampliar o entendimento dos complexos mecanismos relacionados à ocorrência desse evento, que pode originar piores resultados em saúde e implicar aumento da utilização de cuidados e dos custos em saúde.

Outros estudos também devem ser realizados para se conhecer os motivos de descontinuidade e de troca da terapia dessa população com objetivo de subsidiar o desenvolvimento de ações que visem ao maior benefício do tratamento.

$\mathrm{O}$ entendimento de fatores relacionados à não adesão com os biológicos nas doenças reumáticas é necessário como primeiro passo para intervenções efetivas com o intuito de aumentar os comportamentos de adesão e diminuir o risco de respostas terapêuticas inadequadas nessa população. 


\section{Resumen}

Este estudio tuvo como meta estimar e investigar los factores asociados a la falta de adherencia en el tratamiento con medicamentos biológicos etanercept y adalimumab en pacientes con enfermedades reumáticas, tratados por el Sistema Único de Salud de Minas Gerais, Brasil. Se realizó un estudio prospectivo no concurrente, basado en los registros de ventas en farmacias de adultos al comienzo del tratamiento. Se estimó la no adherencia mediante el método de la proporción de días cubierto (PDC) y se consideraron los pacientes no adherentes con $P D C<0,8$. Se identificaron a 1.150 pacientes, de los cuales el 64,3\% sufría artritis reumatoide, el 8,5\% artritis psoriásica y el 27,2\% con espondilitis anquilosante. La no-adhesión fue de un 33,5\% y los factores asociados fueron: edad de 1939 años; diagnóstico de artritis reumatoide; mujer; tratamiento inicial con etanercept y cambio de medicamento. Los resultados indican una alta falta de adherencia, lo que puede conllevar a peores resultados de salud y resultar en el uso de cuidados especializados y suponer costes mayores para el servicio de salud.

Cumplimiento de la Medicación; Terapia Biológica; Enfermedades REumáticas

\section{Colaboradores}

C. G. S. Dabés foi responsável pela concepção, planejamento, análise e interpretação dos dados, redação do artigo e revisão crítica do conteúdo. A. M. Almeida colaborou na revisão da análise e interpretação dos dados, redação do artigo e revisão crítica do conteúdo. F. A. Acurcio contribuiu na concepção, planejamento e revisão crítica do conteúdo.

\section{Agradecimentos}

À Secretaria Estadual de Saúde de Minas Gerais. Aos colegas da Pós-graduação em Medicamentos e Assistência Farmacêutica da Faculdade de Farmácia, Universidade Federal de Minas Gerais.

\section{Referências}

1. Marques-Neto JF, Gonçalves ET, Langen LFOB, Cunha MFL, Radominski S, Oliveira SM, et al. Estudo multicêntrico da prevalência da artrite reumatoide do adulto em amostras da população brasileira. Rev Bras Reumatol 1993; 33:169-73.

2. Senna ER, Barros AL, Silva EO, Costa IF, Pereira LV, Ciconelli RM, et al. Prevalence of rheumatic diseases in Brazil: a study using the COPCORD approach. J Rheumatol 2004; 31:594-7.

3. Dhir V, Aggarwal A. Psoriatic arthritis: a critical review. Clin Rev Allergy Immunol 2013; 44:141-8.

4. Braun J, Sieper J. Ankylosing spondylitis. Lancet 2007; 369:1379-90.

5. Bonafede M, Fox KM, Watson C, Princic N, Gandra SR. Treatment patterns in the first year after initiating tumor necrosis factor blockers in real-world settings. Adv Ther 2012; 29:664-74.

6. Curkendall S, Patel V, Gleeson M, Campbell RS, Zagari M, Dubois R. Compliance with biologic therapies for rheumatoid arthritis: do patient outof-pocket payments matter? Arthritis Rheum 2008; 59:1519-26.

7. van den Bemt BJF, Zwikker HE, van den Ende CHM. Medication adherence in patients with rheumatoid arthritis: a critical appraisal of the existing literature. Expert Rev Clin Immunol 2012; 8:337-51.

8. World Health Organization. Adherence to longterm therapies: evidence for action. Geneva: World Health Organization; 2003.

9. Ministério da Saúde. Portaria SCTIE no 66, de 6 de novembro de 2006. Aprova o Protocolo Clínico e Diretrizes Terapêuticas - artrite reumatoide. Diário Oficial da União 2006; 10 nov.

10. Peterson AM, Nau DP, Cramer JA, Benner J, Gwadry-Sridhar F, Nichol M. A checklist for medication compliance and persistence studies using retrospective databases. Value Health 2007; 10: 3-12.

11. Li P, Blum MA, Von Feldt J, Hennessy S, Doshi JA. Adherence, discontinuation, and switching of biologic therapies in medicaid enrollees with rheumatoid arthritis. Value Health 2010; 13:805-12.

12. Borah BJ, Huang X, Zarotsky V, Globe D. Trends in RA patients' adherence to subcutaneous antiTNF therapies and costs. Curr Med Res Opin 2009; 25:1365-77.

13. Harley CR, Frytak JR, Tandon N. Treatment compliance and dosage administration among rheumatoid arthritis patients receiving infliximab, etanercept, or methotrexate. Am J Manag Care 2003; 9 (6 Suppl):S136-43.

14. Tang B, Rahman M, Waters HC, Callegari P. Treatment persistence with adalimumab, etanercept, or infliximab in combination with methotrexate and the effects on health care costs in patients with rheumatoid arthritis. Clin Ther 2008; 30:1375-84.

15. Ministério da Saúde. Portaria no 2.981, de 26 de novembro de 2009. Aprova o Componente Especializado da Assistência Farmacêutica. Diário Oficial da União 2009; 30 nov. 
16. Dhir V, Aggarwal A. Psoriatic arthritis: a critical review. Clin Rev Allergy Immunol 2013; 44:141-8.

17. Duclos M, Gossec L, Ruyssen-Witrand A, Salliot C, Luc M, Guignard S, et al. Retention rates of tumor necrosis factor blockers in daily practice in 770 rheumatic patients. J Rheumatol 2006; 33:2433-8.

18. Titton DC, Silveira IG, Louzada-Junior P, Hayata AL, Carvalho HMS, Ranza R, et al. Registro Brasileiro de Biológicos: processo de implementação e resultados preliminares do BiobadaBrasil. Rev Bras Reumatol 2011; 51:152-60.

19. Wiens A, Grochocki MC, Pontarolli DRS, Venson R, Correr CJ, Pontarolo R. Perfil dos usuários de anticitocinas disponibilizadas pelo Sistema Único de Saúde no estado do Paraná para o tratamento da artrite reumatoide. Rev Bras Reumatol 2012; 52:203-13.

20. Campos Neto OH, Acurcio FA, Machado MA, Ferré F, Barbosa FL, Cherchiglia ML, et al. Médicos, advogados e indústria farmacêutica na judicialização da saúde em Minas Gerais, Brasil. Rev Saúde Pública 2012; 46:784-90.

21. Gallinaro AL, Ventura C, Barros PDS, Gonçalves CR. Espondiloartrites: análise de uma série brasileira comparada a uma grande casuística iberoamericana (estudo RESPONDIA). Rev Bras Reumatol 2010; 50:581-9.

22. Bakland G, Nossent HC. Epidemiology of spondyloarthritis: a review. Curr Rheumatol Rep 2013; 15:351.

23. Grijalva CG, Chung CP, Arbogast PG, Stein CM, Mitchel Jr. EF, Griffin MR. Assessment of adherence to and persistence on disease-modifying antirheumatic drugs (MMCDs) in patients with rheumatoid arthritis. Med Care 2007; 45(10 Suppl 2):S66-76.

24. Fidder HH, Singendonk MM, van der Have M, Oldenburg B, van Oijen MG. Low rates of adherence for tumor necrosis factor- $\alpha$ inhibitors in Crohn's disease and rheumatoid arthritis: results of a systematic review. World J Gastroenterol 2013; 19:4344-50.

25. Arturi P, Schneeberger EE, Sommerfleck F, Buschiazzo E, Ledesma C, Maldonado Cocco JA, et al. Adherence to treatmet in patients with ankylosing spondylitis. Clin Rheumatol 2013; 32:1007-15.

26. Pasma A, van't Spijker A, Hazes JM, Busschbach JJ, Luime JJ. Factors associated with adherence to pharmaceutical treatment for rheumatoid arthritis patients: a systematic review. Semin Arthritis Rheum 2013; 43:18-28.

27. Arends S, Brouwer E, van der Veer E, Groen H, Leijsma MK, Houtman PM, et al. Baseline predictors of response and discontinuation of tumor necrosis factor-alpha blocking therapy in ankylosing spondylitis: a prospective longitudinal observational cohort study. Arthritis Res Ther 2011; 13:R94.
28. Fénix-Caballero S, Alegre-del Rey EJ, Castaño-Lara R, Puigventós-Latorre F, Borrero-Rubio JM, LópezVallejo JF. Direct and indirect comparison of the efficacy and safety of adalimumab, etanercept, infliximab and golimumab in psoriatic arthritis. J Clin Pharm Ther 2013; 38:286-93.

29. Machado MA, Barbosa MM, Almeida AM, Araújo VE, Kakehasi AM, Andrade EI, et al. Treatment of ankylosing spondylitis with TNF blockers: a metaanalysis. Rheumatol Int 2013; 33:2199-213.

30. Aaltonen KJ, Virkki LM, Malmivaara A, Konttinen YT, Nordström DC, Blom M. Systematic review and meta-analysis of the efficacy and safety of existing TNF blocking agents in treatment of rheumatoid arthritis. PLoS One 2012; 7:e30275.

31. Singh JA, Christensen R, Wells GA, Suarez-Almazor ME, Buchbinder R, Lopez-Olivo MA, et al. Biologics for rheumatoid arthritis: an overview of Cochrane reviews. Cochrane Database Syst Rev 2009; (4):CD007848.

32. Claxton AJ, Cramer J, Pierce C. A systematic review of the associations between dose regimens and medication compliance. Clin Ther 2001; 23: 1296-310.

33. Huynh TK, Ostergaard A, Egsmose C, Madsen OR. Preferences of patients and health professionals for route and frequency of administration of biologic agents in the treatment of rheumatoid arthritis. Patient Prefer Adherence 2014; 8:93-9.

34. Carmona L, Gómez-Reino JJ; BIOBADASER Group. Survival of TNF antagonists in spondylarthritis is better than in rheumatoid arthritis. Data from the Spanish registry BIOBADASER. Arthritis Res Ther 2006; 8:R72.

35. Heiberg MS, Koldingsnes W, Mikkelsen K, Rødevand E, Kaufmann C, Mowinckel P, et al. The comparative one-year performance of anti-tumor necrosis factor alpha drugs in patients with rheumatoid arthritis, psoriatic arthritis, and ankylosing spondylitis: results from a longitudinal, observational, multicenter study. Arthritis Rheum 2008; 59:234-40.

36. Mok CC, Chan KY, Lee KL, Tam LS, Lee KW. Factors associated with withdrawal of the anti-TNF $\alpha$ biologics in the treatment of rheumatic diseases: data from the Hong Kong Biologics Registry. Int J Rheum Dis 2014; 17 Suppl 3:1-8.

37. Sattler EL, Lee JS, Perri M. Medication (re)fill adherence measures derived from pharmacy claims data in older Americans: a review of the literature. Drugs Aging 2013; 30:383-99.

38. Iuga AO, McGuire MJ. Adherence and health care costs. Risk Manag Healthc Policy 2014; 7:35-44.

Recebido em 12/Nov/2014

Versão final reapresentada em 16/Abr/2015

Aprovado em 03/Jun/2015 